\title{
An RCT of treatment for idiopathic membranous nephropathy
}

Good evidence for the best approach to reduce renal function decline in patients with progressive, idiopathic membranous nephropathy is lacking. A new randomized controlled trial (RCT) conducted across 37 renal units in the UK has now demonstrated that a regimen of prednisolone plus chlorambucil is superior to ciclosporin and supportive therapy in reducing the rate of renal function decline in these patients.

Membranous nephropathy has a variable natural history, but approximately $20 \%$ of patients will progress to end-stage renal disease. Peter Mathieson initiated the current trial in the 1990 s to address the issue he considered to be most important in membranous nephropathy - the best treatment approach for severely affected patients with deteriorating kidney function.

Mathieson and colleagues randomly assigned 108 patients with idiopathic membranous nephropathy and deteriorating renal function to receive either supportive therapy (including renin-angiotensin-system blockers, statins and anticoagulants), supportive therapy plus 6 months of prednisolone

$$
\text { and chlorambucil, or }
$$
supportive therapy plus 12 months of ciclosporin. Of 106 patients with available follow-up data, significantly fewer patients in the prednisolone and chlorambucil group than in the supportive therapy group reached the primary end point of a $20 \%$ decline in excretory renal function (58\% of patients versus $84 \%$ of patients, respectively; HR 0.44, 95\% CI 0.24-0.78). By contrast, no difference was observed in the primary end point between the ciclosporin and supportive therapy groups (HR 1.17, 95\% CI 0.7-1.95).

The researchers recorded 390 adverse avents, 117 of which were considered to be serious. 56 of these serious adverse events occurred in the prednisolone plus chlorambucil group versus 24 in the supportive therapy group $(P=0.048)$. "The high rate of shortterm adverse events in all three groups shows that this subset of patients is subject to major morbidity irrespective of treatment," notes Mathieson. "However, as serious adverse events were most frequent in the prednisolone plus chlorambucil group, the benefits of this therapy must be balanced against the risk of adverse events."

The researchers say that recent advances, including the description of anti-PLA2R1 autoantibodies in patients with idiopathic membranous nephropathy, highlight important future possibilities for better diagnosis and monitoring. "Future RCTs should compare modern treatments that have fewer short-term adverse effects with the prednisolone plus chlorambucil combination, which is the most potent treatment tested so far," says Mathieson.

Susan J. Allison

Original article Howman, A. et al. Immunosuppression for progressive membranous nephropathy: a UK randomised controlled trial. Lancet doi:10.1016/S01406736(12)61566-9

Further reading Ruggenenti, P. \& Remuzzi, G. Idiopathic membranous nephropathy: back to the future? Lancet doi:10.1016/S0140-6736(12)62033-9 